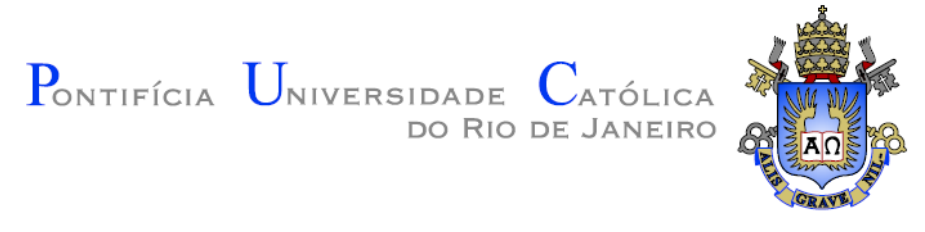

Rodrigo Marques Almeida da Silva

\author{
Um Método Otimizado de Renderização \\ Fotorealista com Distribuição Estatística e \\ Seleção Automática de Técnicas
}

Tese de Doutorado

Tese apresentada como requisito parcial para obtenção do grau de Doutor pelo Programa de Pós-graduação em Informática do Departamento de Informática da PUC-Rio

Orientador: Prof. Bruno Feijó 
Pontifícia Universidade Católica $_{\text {a }}$ DO RIO DE JANEIRO

Rodrigo Marques Almeida da Silva

\section{Um Método Otimizado de Renderização Fotorealista com Distribuição Estatística e Seleção Automática de Técnicas}

Tese apresentada como requisito parcial para obtenção do grau de Doutor pelo Programa de Pós-graduação em Informática do Departamento de Informática da PUC-Rio do Centro Técnico Científico da PUC-Rio. Aprovada pela Comissão Examinadora abaixo assinada.

Prof. Bruno Feijó Orientador

Departamento de Informática - PUC-Rio

Prof. Hélio Cortes Vieira Lopes

Departamento de Informática - PUC-Rio

Prof. Markus Endler Departamento de Informática - PUC-Rio

Prof. Soraia Raupp Musse

Departamento de Informática - PUC-RS

Prof. Luiz Eduardo Azambuja Sauerbronn

Departamento de Informática - UFRJ

Prof. José Eugênio Leal Coordenador(a) Setorial do Centro

Técnico Científico - PUC-Rio

Rio de Janeiro, 26 de março de 2015 
Todos os direitos reservados. É proibida a reprodução total ou parcial do trabalho sem autorização da universidade, do autor e do orientador.

\section{Rodrigo Marques Almeida da Silva}

Graduou-se em Engenharia de Computação pela Universidade Federal do Espírito Santo em 2008, obteve o título de Mestre em Informática pela PUC-Rio em 2010. Desde 2009 trabalha no VisionLab desenvolvendo sistemas de realidade virtual e aumentada e visualização científica, e desde 2010 trabalha como Pesquisador na equipe de P\&D em Efeitos Visuais do Grupo Globo.

Ficha Catalográfica

Silva, Rodrigo Marques Almeida da

Um método otimizado de renderização fotorealista com distribuição estatística e seleção automática de técnicas / Rodrigo Marques Almeida da Silva ; orientador: Bruno Feijó. -2015 .

180 f. : il. (color.) ; $30 \mathrm{~cm}$

Tese (doutorado)-Pontifícia Universidade Católica do Rio de Janeiro, Departamento de Informática, 2015.

Inclui bibliografia

1. Informática - Teses. 2. Renderização Fotorealista. 3. Fila de Renderização. 4. Traçado de Raios. 5. Computação em Nuvem. 6. Árvores de Shade. I. Feijó, Bruno. II. Pontifícia Universidade Católica do Rio de Janeiro. Departamento de Informática. III. Título. 
A meu pai (in memorian), a minha mãe, minha esposa, meus irmãos (inclui Pablo), meus avós e tios e primos. 


\section{Agradecimentos}

Ao meu pai, Clésio Marques da Silva (in memoriam), que sempre apostou no estudo e me proporcionou a força e a vontade de realizar um objetivo tão grande.

À minha família e amigos, pelo grande apoio, sem os quais este trabalho não poderia ter sido realizado.

À minha esposa Ana Paula Sperandio, por sempre me dar força para lutar.

Aos companheiros de luta, em especial a Pablo Bioni, irmão que me apoiou desde o início nessa jornada.

Ao meu orientador Bruno Feijó, pela grande dedicação, apoio e incentivo à pesquisa, sem o qual, esse trabalho não seria possível.

Ao VisionLab/PUC-Rio pelos auxílios concedidos e as oportunidades dentro do projeto com a TV Globo.

À TV Globo e, em destaque, ao departamento de P\&D em VFX, pelo suporte e acesso a equipamentos especiais, sem os quais o trabalho não teria alcançado ganho prático.

Ao CNpQ pelo apoio financeiro.

À FINEP pelas facilidades de infraestrutura na PUC-Rio. 


\section{Resumo}

Silva, Rodrigo Marques Almeida da; Feijó, Bruno (Orientador). Um Método Otimizado de Renderização Fotorealista com Distribuição Estatística e Seleção Automática de Técnicas. Rio de Janeiro, 2015. 180p. Tese de Doutorado - Departamento de Informática, Pontifícia Universidade Católica do Rio de Janeiro.

O processo de renderização fotoreal para cinema e TV demanda, cada vez mais, poder de processamento, necessitando não só de algoritmos paralelos, bem como sistema de distribuição de tarefas. No entanto, mesmo em sistemas de produção, o tempo necessário para avaliar uma animação pode chegar a vários dias, dificultando a melhoria da qualidade artística e limitando alterações. Neste trabalho foca-se na otimização de três processos inerentes à renderização, a renderização local, na qual o sistema trabalha para renderizar um conjunto de pixels de forma otimizada, aproveitando os recursos de hardware disponíveis e aproveitando dados de renderizações previamente realizadas, pelo nó ou teste. O processo de gerenciamento, extremamente crítico para o resultado, é alterado para não só distribuir, mas analisar toda a infraestrutura de renderização, otimizando o processo de distribuição e permitindo o estabelecimento de metas como prazo e custo. Além disso, o modelo de gerenciamento é expandido para a nuvem, utilizando-a como transbordo de processamento. Ainda, um novo processo foi criado para avaliar a renderização de forma colaborativa, onde cada nó comunica resultados parciais e assim otimiza a renderização de outros. Por fim, diversas técnicas inovadoras foram criadas para melhorar o processo como um todo, removendo desperdícios e reaproveitando trabalho.

\section{Palavras-chave}

Renderização Fotorealista; Fila de Renderização; Traçado de Raios; Computação em Nuvem; Árvores de Shade 


\section{Abstract}

Silva, Rodrigo Marques Almeida da; Feijó, Bruno (Advisor). An Optimized Photorealistic Rendering Method with Statistic Distribution and Automatic Rendering Technique Selection. Rio de Janeiro, 2015. 180p. DSc. Thesis - Departamento de Informática, Pontifícia Universidade Católica do Rio de Janeiro.

The photorealistic rendering process for cinema and TV increasingly demands processing power, requiring fast parallel algorithms and effective task distribution systems. However, the processes currently used by the academia and by the industry still consume several days to evaluate an animation in super-resolution (typically $8 \mathrm{~K}$ ), what makes difficult the improvement of artistic quality and limits the number of experiments with scene parameters. In this work, we focus on the optimization of three processes involved in photorealistic rendering, reducing the total time of rendering substantially. Firstly, we optimize the local rendering, in which the system works to render a set of pixels optimally, taking advantage of the available hardware resources and using previous rendering data. Secondly, we optimize the management process, which is changed not only to distribute frames but also to analyze all the rendering infrastructure, optimizing the distribution process and allowing the establishment of goals as time and cost. Furthermore, the management model is expanded to the cloud, using the cloud as a processing overflow. Thirdly, we propose a new optimized process to evaluate the rendering task collaboratively, where each node communicates partial results to other nodes, allowing the optimization of the rendering process in all nodes. Altogether, this thesis is an innovative proposal to improve the whole process of high-performance rendering, removing waste of resources and reducing rework.

\section{Keywords}

Photorealistic Rendering; Render Queue; Ray Tracing; Cloud Computing; Shade Tree 


\section{Sumário}

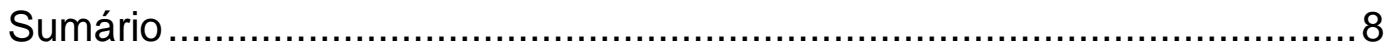

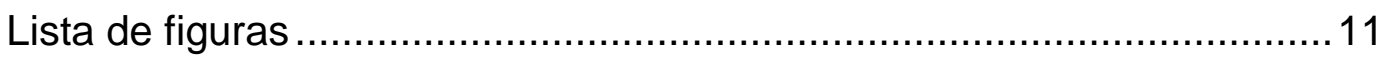

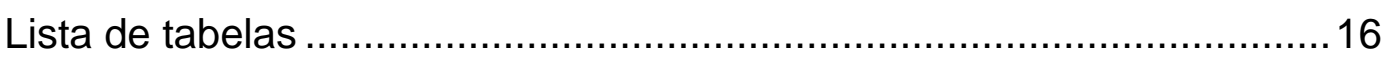

Lista de equações ............................................................................ 17

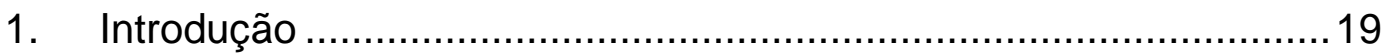

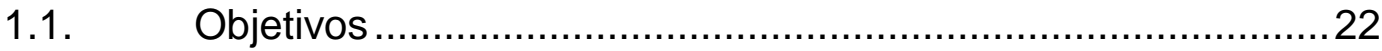

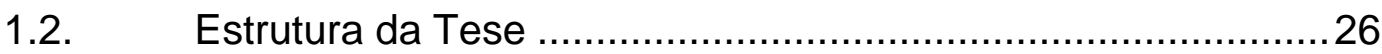

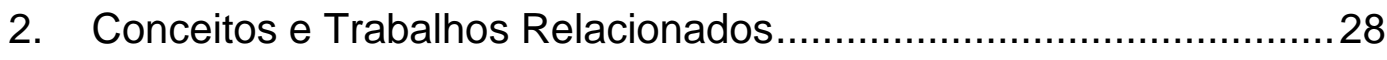

2.1 Elementos Básicos para Renderização Fotorealista................28

2.2 Aceleração de Renderização em Alta Resolução …………......33

2.3 Metodologia e Gerenciamento de Renderização ...................... 41

3. Otimização em Nível de Execução ................................................48

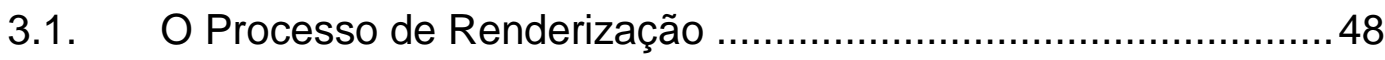

3.1.1. Complexidade Geométrica.................................................

3.1.2. Complexidade de Material e Luzes ........................................52

3.1.3. Subdivisão baseada em custo …………….........................54

3.1.4. Aproveitamento de Custos ...................................................56

3.1.5. Otimização na Renderização ……………………………..... 62

3.1.6. Otimização de Shade Tree ....................................................63

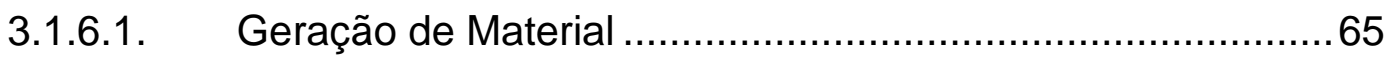

3.1.7. Renderização Multitécnica ................................................... 69 
3.1.7.1. Combinação de Técnicas ………………...........................73

3.1.8. Cache

3.2. Arquitetura do Renderizador ................................................

3.2.1. Rotina Principal do Renderizador ......................................... 81

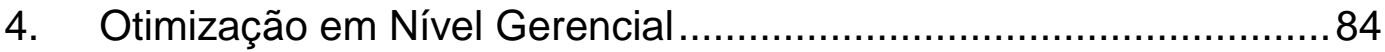

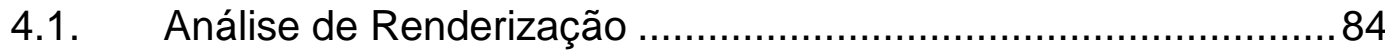

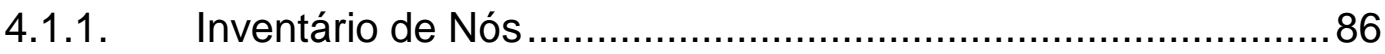

4.2. Arquitetura de Renderização em Cluster ...................................87

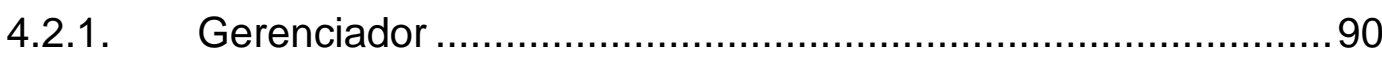

4.2.2. Processo de Inicialização de Renderização..............................92

4.2.3. Distribuição sem restrição temporal ........................................94

4.2.4. Distribuição com restrição temporal ........................................98

4.3. Arquitetura de Renderização em Nuvem ................................. 103

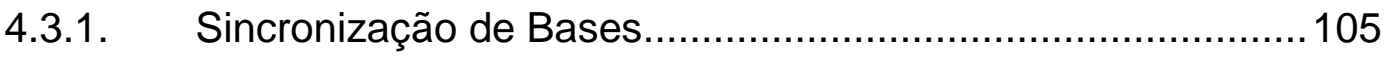

4.3.2. Distribuição com restrição temporal ....................................107

4.3.3. Distribuição com restrição de custos ...................................110

4.4. Múltiplos Trabalhos ................................................................112

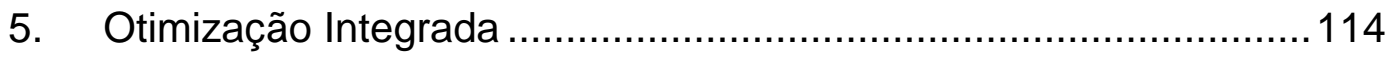

5.1. Compartilhamento de Informações ……………......................115

5.1.1. Método em Pré-Calculo .....................................................117

5.1.2. Método em Execução ………………………………........118

5.1.3. Dump de dados................................................................ 119

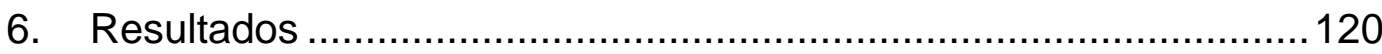

6.1. Metodologia de Teste de Desempenho...................................120

6.2. Metodologia de Análise Estatística .........................................127 


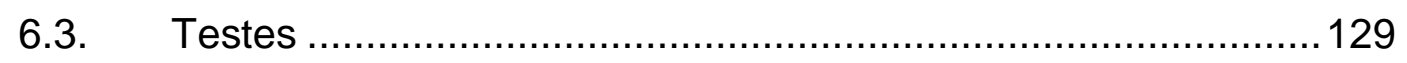

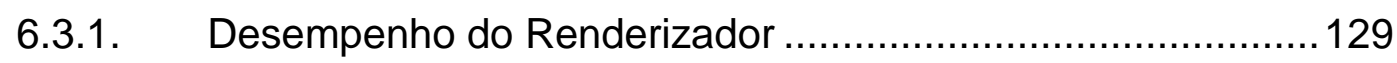

6.3.2. Qualidade de Redução de Técnica .......................................139

6.3.3. Desempenho do Gerenciador ............................................. 141

6.3.4. Desempenho do Gerenciador com Colaboração ....................147

6.3.5. Desempenho de Transbordo para Nuvem ...........................150

6.3.6. Desempenho GPU x CPU................................................... 153

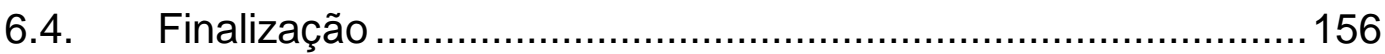

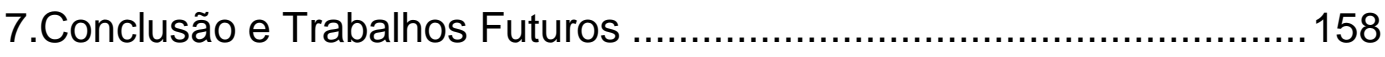

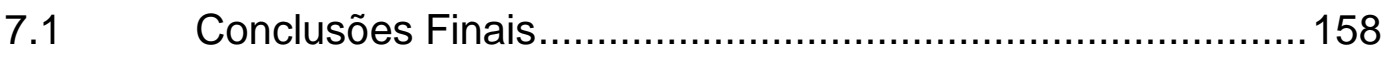

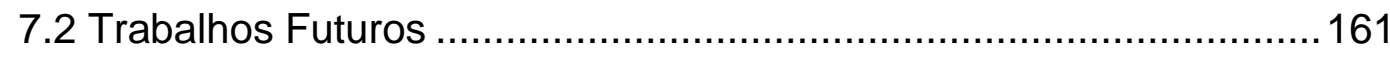

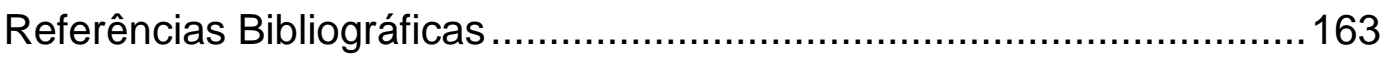

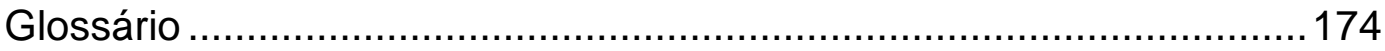




\section{Lista de figuras}

Figura 1: A referência “Utah Fairy"(Wald,I. 2010) nas versões:

(a) ray tracing em tempo real , $180 \mathrm{~K}$ triângulos, $1024 \times 1024$, 1 única fonte de luz e aprox. 4 fps em dual core 64 bits (figura extraída de Wald et al., 2007);

(b) ray tracing de imagem HDTV, $1280 \times 720$, wide-screen, com desfocagem e motion blur, aprox. 6M micropolígonos, aprox. 60 seg em GPU (para um único frame)

(figura extraída de Hou et al., 2010).

Figura 2: Cena complexa com qualidade de cinema, com 678 milhões de triângulos, 106 minutos de rendering, em um Apple G5 com 2 processadores PowerPC de $2 \mathrm{GHz}$ (figura extraída de Christensen et al. (2006)) .21

Figura 3: Inter-reflexão com uso de ray tracing no filme "Cars (Pixar, 2006)": (a) reflexo dos olhos e vidro frontal ;

(b) reflexão entre partes cromadas. Figura composta a partir de Christensen et al. (2006) 30

Figura 4: Sombras bem definidas e detalhadas geradas por ray tracing, do filme "Cars (Pixar, 2006)" contendo 1000 fontes de luz (figura extraída de Christensen et al. (2006))

Figura 5: Oclusão de ambiente gerado por ray tracing em imagens de alta qualidade (figura extraída de Christensen et al. (2006)). 32

Figura 6: Exemplo da utilização do Fakeosity (exemplo produzido). .33

Figura 7: Comparação entre o resultado do RayTracing e Virtual Point Light a) resultado obtido com ray tracing com iluminação global. b) resultado obtido com VPL. (Imagens extraídas de Walter et al (2012))

Figura 8: Virtual Point Light simula a influencia de uma luz através de pontos adicionais de luz, já o Virtual Ray Light utiliza uma interpolação de todo o trajeto entre os 
pontos para simular a interação, sendo assim, muito

mais denso. (imagem de Novàk et al (2012)).

Figura 9: Estruturas Hierárquicas:

a) Grade Regular (Fernando, 2004)

b) Kd-Tree (exemplo produzido) c) Octree (Pharr, 2005)

Figura 10: Sombras suaves utilizando amostragem

de Monte Carlo (a) baixa amostragem (b) alta amostragem

(imagens produzidas)

Figura 11: Shade tree e código correspondente na

RenderMan (Pixar, 1970)

Figura 12: Sistema de criação de Shade Trees desenvolvido

nesta pesquisa

Figura 13: Espectro de cor visível e o gamut de câmeras

e televisores (Gaggioni et al, 2012)

Figura 14: Workflow de Renderização (Rasterização)

(imagem produzida)

Figura 15: Problemas na subdivisão da renderização.

Cena do Maracanã, gerado pelo autor para a novela

Avenida Brasil (TV Globo, 2012). Imagem reproduzida

sob a política de "fair use".

Figura 16: Projeção de nós da BVH (imagem produzida)

Figura 17: Subdivisão utilizada por Abraham et al (2004)

Figura 18: Subdivisão utilizada pelo sistema

(imagem produzida (algoritmo))

Figura 19: Imagem de Performance: cada pixel de um

frame tem as informações de desempenho armazenadas

(\#Rays, CPU Time, \#MC Samples, Stack Size,

\#Texture Samples, Memory, \#Ray misses). Estas informações

permitem a subdivisão otimizada do frame (exemplificada

com as opções Quadtree e kd-tree).

(imagem produzida (algoritmo))

Figura 20: Compensação da Imagem de Performance

(imagem produzida (algoritmo)) 
Figura 21: Sistema de Criação de Shade Trees Desenvolvido ...............64

Figura 22: Criação de Bloco Composto..........................................66

Figura 23: Algoritmo de Geração de Material (image produzida) ...........67

Figura 24: Diferenças entre técnicas, usando a

cena "San Miguel" (Barringer R. et al, 2014) ......................................71

Figura 25: Blend Zones (imagem produzida) ...................................73

Figura 26: Múltiplas técnicas técnicas usadas em

uma cena de Saramandaia (TV Globo, 2014). Imagem

reproduzida sob a política de fair use ............................................... 74

Figura 27: Irradiance Cache (Krivanek et al., 2009) .............................75

Figura 28: Amostragem dos Irradiance Caches

(Krivanek et al., 2009) ...................................................................... 76

Figura 29: Arquitetura do sistema local proposto

(imagem produzida)

Figura 30: Modelo básico de dados do banco de dados

(imagem produzida)

Figura 31: Interface MaxScript, usando a cena

"San Miguel" (Barringer R. et al, 2014).

Figura 32: Fluxograma de operação do sistema proposto ......................82

Figura 33: Processo convencional de distribuição de frames .................85

Figura 34 : Arquitetura de alto nível do Gerenciador e

do Sistema de Controle dos Nós (imagem produzida)

Figura 35: Interface Web do Gerenciador

(imagem do sistema)

Figura 36: Estrutura de Dados do Servidores SQL

(imagem produzida)

Figura 37: Campos de Submissão (imagem do sistema)

Figura 38: Inicialização da renderização de cenas

(imagem produzida)

Figura 39: Classificação e categorização de frames

(imagem produzida)

Figura 40: Ordem de execução do bloco de frames (imagem produzida) 
Figura 41: Fluxo de gerenciamento dos frames (sem restrições) (imagem produzida) .98

Figura 42: a) Esquemático do algoritmo de subdivisão

de frame. b) Imagem de Performance sendo subdividida

pelo BSP. c) Máscaras de renderização (branco habilita renderização, preto impede renderização)

Figura 43: Algoritmo de gerenciamento com restrição temporal (imagem produzida) 102

Figura 44: Arquitetura convencional de transbordo para a nuvem ..........104

Figura 45: Cloudberry, sistema de sincronização de bases.

Figura 46: Processo de distribuição na nuvem com restrição temporal.

Figura 47: Alocação de nós com restrição de custo (imagem produzida)

Figura 48: Exemplo de escalonamento usando o EDF -

Earliest Deadline First, extraído de (Tanenbaum et al, 2004)

Figura 49: Grafo de frames proposto para a

renderização colaborativa (imagem produzida)

Figura 50: Intervalo de análise em execução

(imagem produzida)

Figura 51: Arquitetura de testes usando o Deadline

e o MPI do HPCPack (imagem produzida).

Figura 52: Cenas do teste a) cena "San Miguel"

(Barringer R. et al, 2014) b) Cena do Maracanã, gerado

pelo autor para a novela Avenida Brasil (TV Globo, 2012).

Imagem reproduzida sob a política de "fair use".

Figura 53: Opções de linha de comando do

sistema de avaliação

Figura 54: Hardware empregado nos testes, cedidos

pela TV Globo para testes. a) Intel Xeon Phi

b) Nós de renderização c) Storage Equalogic e

NVidia VCA

Figura 55: Resultado Gráfico de Frames - Teste 1.1 
Figura 56: Resultado Gráfico de Frames - Teste 1.2 .......................... 131

Figura 57: Resultado Gráfico de Frames - Teste 2.1 ......................... 132

Figura 58: Resultado Gráfico de Frames - Teste 2.2 .......................... 133

Figura 59: Resultado Gráfico de Frames - Teste 2.3.......................... 134

Figura 60: Resultado Gráfico de Frames - Teste 2.4 .......................... 135

Figura 61: Resultado Gráfico de Frames - Teste 3.1 .......................... 136

Figura 62: Resultado Gráfico de Frames - Teste 3.2......................... 137

Figura 63: Resultado Gráfico de Frames - Teste 4.1 ........................... 140

Figura 64: Resultado Gráfico de Frames - Teste 5.1 ..........................142

Figura 65: Resultado Gráfico de Frames - Teste 5.2........................... 144

Figura 66: Resultado Gráfico de Frames - Teste 6.1 .......................... 145

Figura 67: Resultado Gráfico do Número de Servidores

Alocados (Servidor x Tempo) - Teste 6.1 ........................................ 146

Figura 68: Resultado Gráfico de Frames - Teste 7.1 ...........................146

Figura 69: Resultado Gráfico do Número de Servidores

Alocados (Servidor x Tempo) - Teste 7.1 .......................................... 147

Figura 70: Resultado Gráfico de Frames - Teste 8.1 ........................... 148

Figura 71: Resultado Gráfico de Frames - Teste 9.1 ........................... 149

Figura 72: Resultado Gráfico do Número de Servidores

Alocados (Servidor x Tempo) - Teste 9.1 ......................................... 149

Figura 73: Resultado Gráfico de Frames - Teste 10.1 ......................... 151

Figura 74: Alocação de Servidores - Teste 10.1 ................................ 151

Figura 75: Resultado Gráfico de Frames - Teste 11.1 ......................... 152

Figura 76: Alocação de Servidores - Teste 11.1................................ 153

Figura 77: Resultado Gráfico de Frames - Teste 12.1 ........................ 154

Figura 78: Resultado Gráfico de Frames - Teste 13.1 .........................155 


\section{Lista de tabelas}

Tabela 1: Resultados do tempo de renderização do Teste 1.1 ..............130

Tabela 2: Resultados do tempo de renderização do Teste 1.2 ..............131

Tabela 3: Resultados do tempo de renderização do Teste 2.1 .............132

Tabela 4: Resultados do tempo de renderização do Teste 2.2 ............... 133

Tabela 5: Resultados do tempo de renderização do Teste 2.3 ...............134

Tabela 6: Resultados do tempo de renderização do Teste 2.4 .............. 135

Tabela 7: Resultados do tempo de renderização do Teste 3.1 ..............136

Tabela 8: Resultados do tempo de renderização do Teste 1.2 ..............137

Tabela 9: Resultados Gráficos Teste 1, 2 e 3 -

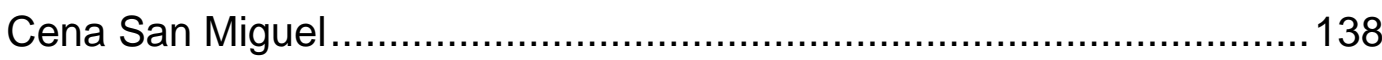

Tabela 10: Resultados Gráficos Teste 1, 2 e 3 -

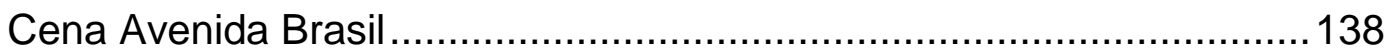

Tabela 11: Resultados do tempo de renderização do Teste 4.1 ............140

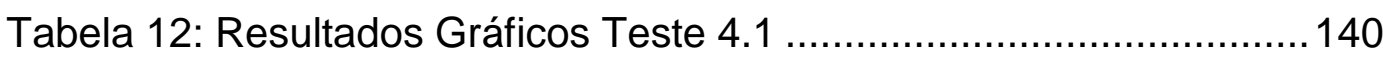

Tabela 13: Resultados do tempo de renderização do Teste 5.1 ............143

Tabela 14: Resultados do tempo de renderização do Teste 5.2 ............. 144

Tabela 15: Resultados do tempo de renderização do Teste 6.1 ............146

Tabela 16: Resultados do tempo de renderização do Teste 7.1 ............147

Tabela 17: Resultados do tempo de renderização do Teste 8.1 ............148

Tabela 18: Resultados do tempo de renderização do Teste 9.1 ............ 149

Tabela 19: Resultados do tempo de renderização do Teste 10.1 ...........151

Tabela 20: Resultados do tempo de renderização do Teste 11.1 ..........153

Tabela 21: Resultados do tempo de renderização do Teste 12.1 .......... 154

Tabela 22: Resultados do tempo de renderização do Teste 13.1 ..........155 


\section{Lista de equações}

Equação 1: Fórmula de custo inicial de um pixel ...................................50

Equação 2: Fórmula de atualização de kgeometria ...............................50

Equação 3: Fórmula de atualização de kmaterial ..................................51

Equação 4: Fórmula de custo geométrico de um pixel.............................52

Equação 5: Fórmula de custo de material de um pixel.............................53

Equação 6: Fatores de Refração e Reflexão.............................................53

Equação 7: Fórmula da computação da imagem

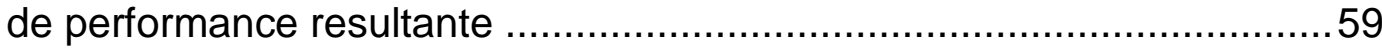

Equação 8: Fórmula de custo de um pixel considerando

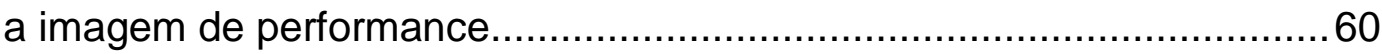

Equação 9: Fórmula da atualização de kperfimagem.............................60

Equação 10: Limite da atualização de kperfimagem .............................61

Equação 11: Fórmula da atualização de kimg .......................................62

Equação 12: Estimativa de Consumo de Memória..................................94

Equação 13: Estimativa de performace dos servidores ..........................97

Equação 14: Estimativa do número de servidores a

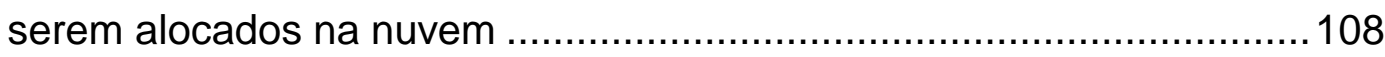

Equação 15: Estimador de tempo por pixel no VRay ............................123

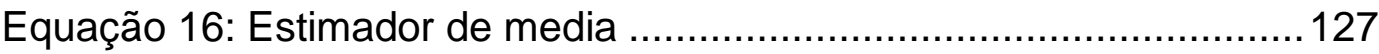

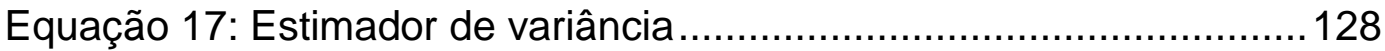

Equação 18: Cálculo do intervalo de confiança.....................................129 
"Há momentos em que a maior sabedoria é parecer não saber nada."

Sun Tzu, A Arte da Guerra. 